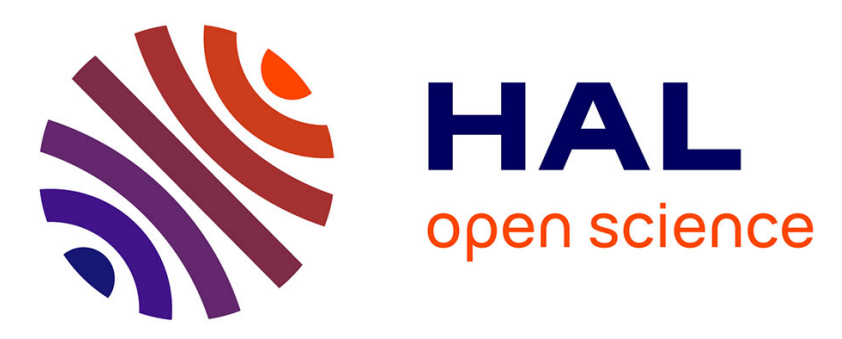

\title{
Peregrine Falcon's Dive: Pullout Maneuver and Flight Control Through Wing Morphing
}

Omar Selim, Erwin Ricky Gowree, Christian Lagemann, Edward Talboys, Chetan Jagadeesh, Christoph Brücker

\section{- To cite this version:}

Omar Selim, Erwin Ricky Gowree, Christian Lagemann, Edward Talboys, Chetan Jagadeesh, et al.. Peregrine Falcon's Dive: Pullout Maneuver and Flight Control Through Wing Morphing. AIAA Journal, 2021, 59 (10), pp.3979-3987. 10.2514/1.J060052 . hal-03407830

\section{HAL Id: hal-03407830 https://hal.science/hal-03407830}

Submitted on 28 Oct 2021

HAL is a multi-disciplinary open access archive for the deposit and dissemination of scientific research documents, whether they are published or not. The documents may come from teaching and research institutions in France or abroad, or from public or private research centers.
L'archive ouverte pluridisciplinaire HAL, est destinée au dépôt et à la diffusion de documents scientifiques de niveau recherche, publiés ou non, émanant des établissements d'enseignement et de recherche français ou étrangers, des laboratoires publics ou privés. 


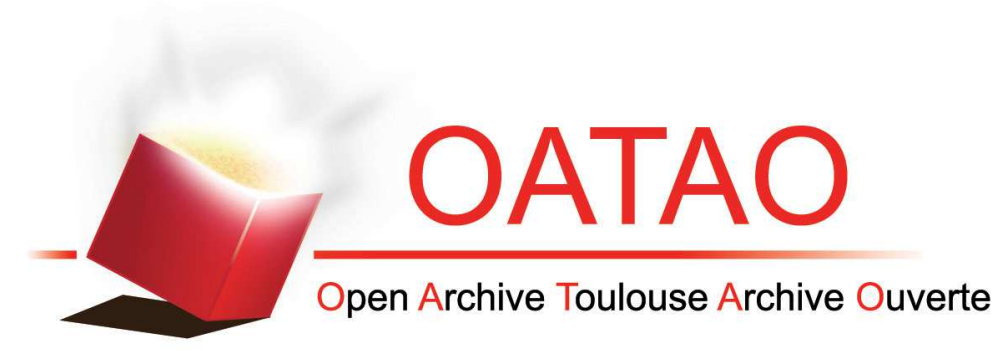

\section{Open Archive Toulouse Archive Ouverte (OATAO)}

OATAO is an open access repository that collects the work of some Toulouse researchers and makes it freely available over the web where possible.

This is an author's version published in: https://oatao.univ-toulouse.fr/28180

Official URL : https://doi.org/10.2514/1.J060052

\section{To cite this version :}

Selim, Omar and Gowree, Erwin Ricky and Lagemann, Christian and Talboys, Edward and Jagadeesh, Chetan and Brücker, Christoph Peregrine Falcon's Dive: Pullout Maneuver and Flight Control Through Wing Morphing. (2021) AIAA Journal. 1-9. ISSN 0001-1452

Any correspondence concerning this service should be sent to the repository administrator: tech-oatao@listes-diff.inp-toulouse.fr 


\title{
Peregrine Falcon's Dive: Pullout Maneuver and Flight Control Through Wing Morphing
}

\author{
Omar Selim* \\ City, University of London, London, England EC1V OHB, United Kingdom \\ Erwin R. Gowree亡 \\ ISAE-SUPAERO, Université de Toulouse, 31055 Toulouse, France \\ Christian Lagemann \\ RWTH Aachen University, 52062 Aachen, Germany \\ and \\ Edward Talboys,, Chetan Jagadeesh, $\mathbb{1}$ and Christoph Brücker** \\ City, University of London, London, England EC1V OHB, United Kingdom
}

https://doi.org/10.2514/1.J060052

\begin{abstract}
During the pullout maneuver, peregrine falcons were observed to adopt a succession of specific flight configurations that are thought to offer an aerodynamic advantage over aerial prey. Analysis of the flight trajectory of a falcon in a controlled environment shows it experiencing load factors up to $3 g$, and further predictions suggest this could be increased up to almost $10 \mathrm{~g}$ during high-speed pullout. This can be attributed to the high maneuverability promoted by lift-generating vortical structures over the wing. Wind-tunnel experiments on life-sized models in the different configurations together with high-fidelity computational fluid dynamics simulations (large-eddy simulations) show that deploying the hand wing in a pullout creates extra vortex lift, which is similar to that of combat aircraft with delta wings. The aerodynamic forces and the position of the aerodynamic center were calculated from the simulations of the flow around the different configurations. This allowed for an analysis of the longitudinal static stability in the early pullout phase, confirming that the falcon is flying unstably in pitch with a positive slope in the pitching moment and a trim angle of attack of about $5 \mathrm{deg}$, which is possibly to maximize responsiveness. The hand wings/primaries were seen to contribute to the augmented stability, acting as "elevons" would on a tailless blended-wing/body aircraft.
\end{abstract}

\begin{tabular}{ll} 
& \multicolumn{1}{c}{ Nomenclature } \\
$A R$ & $=$ aspect ratio, defined as $b^{2} / S$ \\
$b$ & $=$ wingspan, $\mathrm{m}$ \\
$C_{D}$ & $=$ drag coefficient \\
$C_{L}$ & $=$ lift coefficient \\
$C_{L_{p}}$ & $=$ potential lift coefficient \\
$C_{L_{T}}$ & $=$ theoretical lift coefficient \\
$C_{L_{v}}$ & $=$ vortex-lift coefficient \\
$C_{m, \mathrm{cg}}$ & $=$ pitching moment coefficient about center of gravity \\
$C_{m_{0}, \text { ac }}$ & $=$ zero-lift pitching moment coefficient about aerody- \\
$C_{(x, y, z)}$ & $=$ namic center \\
$c$ & $=$ charce coefficient in the $x, y$, or $z$ direction \\
$c_{w}$ & $=$ wing maximum chord, $\mathrm{m}$ \\
$F_{(x, y, z)}$ & $=$ force in the $x, y$, or $z$ direction, $\mathrm{N}$ \\
$g$ & $=$ acceleration of gravity, ms ${ }^{-2}$ \\
$K_{p}$ & $=$ potential flow coefficient
\end{tabular}

Received 10 August 2020; revision received 16 February 2021; accepted for publication 4 May 2021; published online 16 August 2021. Copyright $($ ) 2021 by the American Institute of Aeronautics and Astronautics, Inc. All rights reserved. All requests for copying and permission to reprint should be submitted to CCC at www.copyright.com; employ the eISSN 1533-385X to initiate your request. See also AIAA Rights and Permissions www.aiaa.org/ randp.

*Ph.D. Student, Department of Mechanical Engineering and Aeronautics; omar.selim@city.ac.uk.

${ }^{\dagger}$ Associate Professor, Department of Aerodynamics, Energetics and Propulsion, 10 Avenue Edouard Belin; erwin-ricky.gowree@ isae-supaero.fr.

${ }^{\star}$ Ph.D. Student, Institute of Aerodynamics; c.lagemann@aia.rwth-aachen de.

${ }^{\S}$ Ph.D. Student, Department of Mechanical Engineering and Aeronautics; edward.talboys.1@city.ac.uk.

"Lecturer, Department of Mechanical Engineering and Aeronautics; chetan .jagadeesh.1@city.ac.uk.

**Professor, Department of Mechanical Engineering and Aeronautics; christoph.bruecker@city.ac.uk.

$\begin{array}{lll}K_{v} & =\text { vortex flow coefficient } \\ n & =\text { load factor } \\ q_{\infty} & =\text { freestream dynamic pressure, } \mathrm{Pa} \\ S & =\text { characteristic planform area, } \mathrm{m}^{2} \\ W & =\text { weight, } \mathrm{N} \\ x_{\mathrm{cg}} & =\text { position of center of gravity, } \mathrm{m} \\ \alpha & =\text { angle of attack } \\ \alpha_{t} & =\text { trim angle of attack } \\ \theta & =\text { angle of pitch, rad } \\ \dot{\theta} & =\text { rate of pitch, rad } / \mathrm{s}^{-1} \\ \Lambda & =\text { leading-edge wing sweep }\end{array}$

Subscripts

ac $\quad=$ aerodynamic center

$\mathrm{cg}=$ center of gravity

\section{Introduction}

$\mathbf{T}$ HE peregrine falcon (falco peregrinus) attacks its prey by rapid strike while performing high-speed stoops (dives) due to its ability to undergo a variety of morphological transformation. It climbs to high altitude during thermal soaring with its large wings completely spread, but the most interesting morphological transformation happens during the stoop, shown in Fig. 1. Although the high speed achieved in the teardrop shape [1-3] has so far attracted most of the attention, during the stoop, the falcon also shows additional impressive aerobatic performance in other flight configurations. Teardrop-shape is one of the configurations adopted in a stoop; these different configurations are discussed overleaf. For instance, while readjusting its attitude to increase strike precision, the falcon will open the hand wings, laterally morphing into what is currently designated the cupped shape [1]. This is also employed for slight deceleration. In this configuration, the lateral forces on the wings can be three times the weight of the bird; and peregrine falcons can withstand such high loads due to their superior musculoskeletal 


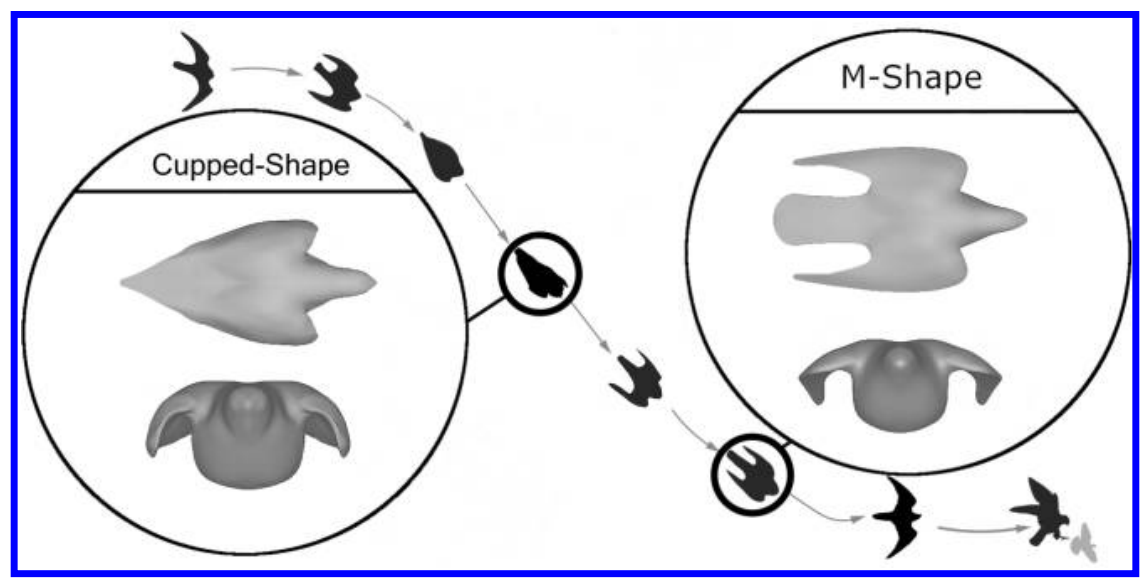

Fig. 1 Morphological transformation of a peregrine falcon at various stages during stoop and pullout. In the bubbles, the elevated and plan views of the corresponding models are shown, which were generated by laser scanning of taxidermy birds and then reproduced in computer-aided design.

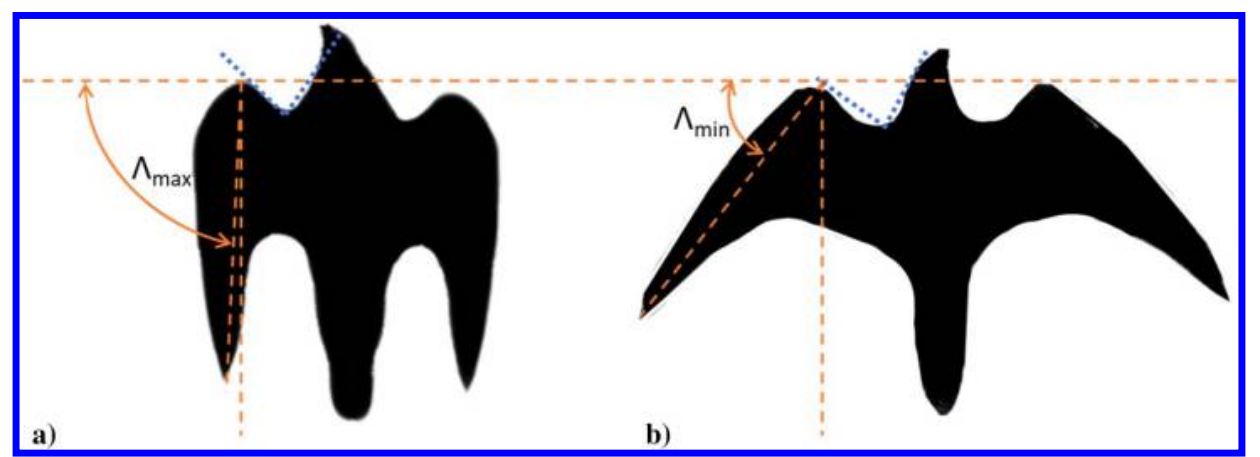

Fig. 2 Schematic of sweep change (orange, dashed lines) from M-shape to open-M configuration, illustrating consistent inboard forward sweep throughout (blue, dashed lines).

structure compared to other birds [4]. In the final phase immediately before or during prey strike, the bird will enter the "pullout," in which it will morph into what is designated the "M shape." In this configuration, the bird is able to generate considerably more lift without the typically associated increase in drag expected for a classical wing, enabling controlled flight at moderately high speeds. These morphological adaptations have been captured on video using sophisticated highspeed recording systems during field experiments [5], and wind-tunnel analysis has provided us with further evidence of the flow behavior that allows the falcon to achieve such impressive maneuverability. This skill is also demonstrated during courtship flights where the falcon can achieve even higher speeds during the dive since it does not have to track prey.

According to Ponitz et al. [5], the falcon's stoop and early pullout can be categorised into a series of characteristic configurations [3]]:

1) The first configuration is the teardrop shape. This is the configuration typically adopted for maximum speed in the beginning of a stoop. It is characterized by the wings being completely retracted into the body and the tail remaining completely furled. The bird has little to no control at this point and is rather similar to an unguided projectile, optimizing speed.

2) The second configuration is the cupped shape. This is the configuration that the bird will adopt in order to adjust its trajectory at high speeds and is accompanied by a marginal deceleration. It is characterized by the trailing edge of the wings remaining attached to the tail and the leading edge morphing laterally away from the body to a position that creates a "cup" of air between them.

3) The third configuration is the open-cupped shape. Somewhat of a bridge between cupped and $M$ shapes, this configuration is seen to be intermittently adopted during a stoop for control purposes. It is characterized similar to the cupped shape but with the

${ }^{\dagger \dagger}$ Private communication with Bleckmann, H., 2015. leading edge further extended, eliminating the cup between the wing and body.

4) The fourth configuration is the $M$ shape. In this configuration, the bird will keep the inboard section of the wing pushed forward while sweeping the outboard section back, vectoring the forces normal to the wing upward, and subsequently increasing the total lift produced. It is characterized by the trailing edge of the wing detaching from the body/tail, a forward sweep in the inboard section, and strong aftsweep in the outboard section, fashioning a shape of the letter "M," shown in Fig. 2a.

5) The fifth configuration is the open-M shape. An extension of the M-shape configuration, this configuration comprises the same inboard characteristics of the $M$ shape while swinging the outboard section (hand wing) to decrease the sweep angle, further increasing the total force. In this configuration, a reduction in aftsweep $\Lambda$ is seen in the outboard section. This typically ranges from $\Lambda_{\min } \approx 40 \mathrm{deg}$ to $\Lambda_{\max } \approx 90 \mathrm{deg}$, as shown in Fig. $2 \mathrm{~b}$.

Each of these phases was observed to not be simply a fixed position in which the bird will robotically morph but rather a smooth, reversible transition between the phases with certain dominating characteristics exhibited. These characteristics are what have been used to categorize the different configurations into which the bird has evolved to morph. This may be for performance optimization, stability augmentation, and/or comfort physiological limits such as maximum shoulder torque [4,6].

Throughout a stoop and pullout maneuver, the falcon will sequence through adopting each of the four configurations mentioned earlier in this paper. During live flight analysis, the bird is seen to adjust its trajectory or correct its attitude very rapidly in the cupped shape and $\mathrm{M}$ shape in order to increase the chances of striking the prey. This is enabled by the excellent roll and yaw control abilities it possesses. What is peculiar is how the tail remains furled with little to no change in a pullout in these recordings, suggesting that the mechanics of such maneuvers are entirely or almost entirely controlled by the wings in that configuration, similar to what delta wings 
can achieve when equipped with elevons at the trailing edge. As opposed to conventional aircraft, the bird does not have a fin and a rudder for lateral control, and therefore uses the wingtips and the tail to achieve these maneuvers. This is confirmed from the live recordings reported in Refs. [7,8], where the bird is seen to open up its wings laterally, sometimes even close to the $\mathrm{M}$ shape during the high-speed dive; however, it tucks them back in into the teardrop shape immediately after to reduce the drag.

Primarily, the falcon's wing morphing when in M-shape and open$M$ configurations serves to vary the amount of lift produced either to reach a specific load factor (and subsequently a specific radius of pullout) or to kill lift in order not to exceed the maximum tolerable bending torque about the bird's shoulder [4,9]. By analyzing the flight trajectory of a falcon in pullout, this paper will strive to calculate the limits of these forces throughout the maneuver and estimate the maximum forces the bird would need to withstand with its superior flight speeds. Secondarily, however, the morphing serves as positional adjustments of the center of pressure: either spanwise in order to reduce the bending torque about the shoulder girdle without sacrificing lift or longitudinally as pitching moment control, augmenting the limited inputs from the furled tail. This fore-and-aft movement due to longitudinal perturbations is not unique to falcons and was observed in hawks and pigeons, but it was often coupled with inputs from a deployed tail $[10,11]$. The geometry and models available for this study were the first four of the five configurations mentioned earlier in this paper. These configurations could be reconstructed from high-resolution multicamera imaging of live-bird flight tests in a previous study by Ponitz et al. [5]. However, the exact geometry of the last "open-M" configuration could not be recovered. Hence, the computational fluid dynamics (CFD) simulation and wind-tunnel test results are limited to those configurations in early pullout. In late pullout, the wing-morphing comprises simple sweep changes taking the configuration from the $\mathrm{M}$ shape to open $\mathrm{M}$. Therefore, we used aerodynamic lifting theory for swept delta wings to extrapolate the performance of the bird in this phase. A direct CFD simulation of that configuration is left for future work because the geometrical data do not exist thus far.

Most fixed-wing/forward thrust unmanned aerial vehicles (UAVs)/ micro aerial vehicles (MAVs) airborne today fly in a statically stable manner. This is due to the benefits of the passive stability: namely, that the aircraft does not require input from the controller or from a flight control computer to respond to small longitudinal perturbations. However, associated with static stability is a limitation of maneuverability. Naturally, a statically stable platform will offer some passive resistance to departure from equilibrium: an undesirable trait of a nimble predator. Conversely, modern fighter aircraft will fly in a statically unstable or neutrally stable configuration so as to exploit the benefits in maneuverability. This kind of flight would not be possible unless controlled by a flight control computer (FCC) able to detect and counter departures from equilibrium instantaneously.

As a continuation of the previous work by Ponitz et al. [5], Gowree et al. [7], and Ponitz et al. [8], the present paper analyzes the aerodynamics and mechanics of the flight of a falcon in the pullout maneuver and the wing morphing. The study focuses on the early pullout phase where the transition is from teardrop in three stages toward $\mathrm{M}$ shape, whereas we use aerodynamic lifting theory to also extend predictions for the open $\mathrm{M}$ in the late phase of pullout. Furthermore, we apply classical flight stability criteria in order to draw parallels with current state-of-the-art highly maneuverable flight demonstrators and to explore the possibility of incorporation of the morphing mechanics and control mechanisms in modern micro air vehicles and unmanned aerial vehicles.

\section{Methods}

\section{A. Life-Size Models}

The geometries of the different wing-morphing states were already used in previous studies and were qualified as good representatives of peregrine falcons in flight $[5,7]$. They were derived from a combination of simultaneous high-resolution digital photographs taken from different perspectives in field experiments and three-dimensional (3D) laser scanning of stuffed animals reconstructed for different flight situations. The scanned geometries were imported into a computeraided design package; and with the help of the detailed flight images [5], the different wing morphologies could be realized with good matches to the images from different perspectives. Physical models were manufactured using 3-D printing techniques for the use in windtunnel studies, and they were coated in a matte black paint in order to increase contrast for the flow visualization. The corresponding 3-D printed models are shown in Fig. 1. The same geometries were exported as STL-type surface meshes (a type of file containing information of a 3-dimensional model exported from computer aided design software) to represent the body in a digital form for the largeeddy simulations (LESs).

\section{B. Simulations}

The computational fluid dynamic simulations were carried out using a commercial software (Simcenter STAR-CCM+), which is able to solve implicit unsteady Reynolds-averaged Navier-Stokes (RANS) and large-eddy simulation calculations. Although not explicitly shown here, the simulation was carried out in three dimensions and on the entire model of the bird. This was due to the importance of the threedimensionality in the flow (as seen in Ref. [7]) and in order to capture adequately the three-dimensional curvature of the wing/body junction and wing twist. In the case of the RANS simulations, a $k$ - $\omega$ shear-stress transport two-equation turbulence model was used; and for the LES, a wall-adapting local-eddy viscosity subgrid-scale model was applied to model the turbulent viscosity. The computational domain was decomposed into near-surface prism layers and a global Cartesian grid with two refinement zones; this can be seen in Fig. 3. The initial mesh was based on an anisotropic hexahedral trimmed grid and had 57 million cells for the M shape, 63 million cells for the cupped shape, and 50 million cells for the teardrop shape. To resolve the fine-scale structures within the boundary layer, a minimum grid spacing of $y^{+} \ll 1$ is used [12]. A second-order-accurate scheme was selected for the temporal and spatial discretization. The initial RANS simulations were regarded as converged when the momentum residuals and the energy dropped below $10^{-5}$. This criterion was reached within 15,000 iterations. The LES corresponded to a physical time of $1 \mathrm{~s}$ where the time step was $\Delta t=10^{-5}$. More information on the stability of the grid and time steps can be found in Ref. [13] and in the supplementary information provided in Ref. [7]. The models used in the simulation were exactly the same as the models that were 3-D printed; therefore, the simulations and experiments could be compared.

In addition to supporting the experimentally measured lift force, the main benefit from the high-fidelity simulations was that it allowed for the calculation of the contribution of a particular section or part of the bird to the overall aerodynamic forces. The forces were categorized into three categories; wing forces (which were split into left and right wings), body forces, and tail forces. This could be achieved by integrating the static pressure and the tangential stress over the corresponding area; and that would give the resulting normal, axial, and side forces of $F_{y}, F_{x}$, and $F_{z}$, respectively. These sectional aerodynamic forces are shown in Fig. 4 (nondimensionalized by dividing by the corresponding sectional planform area).

\section{Wind-Tunnel Test}

The wind-tunnel experiments were conducted with a freestream velocity of $U_{\infty}=22.5 \mathrm{~ms}^{-1}$, which was the speed attained by the falcon while flying at equilibrium during the field experiment at Oleftal Dam in Hellenthal, Germany [5]. This corresponded to a full-scale Reynolds number of $5.8 \times 10^{5}$ based on the length of the bird, and it was replicated during the CFD simulation. Force measurements were carried out in a Göttingen-type wind tunnel at Technische Universität Bergakademie Freiberg in 2013, has a nozzle outlet cross section of $0.30 \mathrm{~m}^{2}(0.6 \times 0.5 \mathrm{~m})$ and a turbulence intensity of $0.04 \%$. The falcon models were placed in the

\footnotetext{
\#Colditz, T., and Gelfert, M., "ProjektarbeitWanderfalke," Student Project, 38 Technische Univ. Bergakademie Freiberg, Freiberg, Germany, 2013.
} 


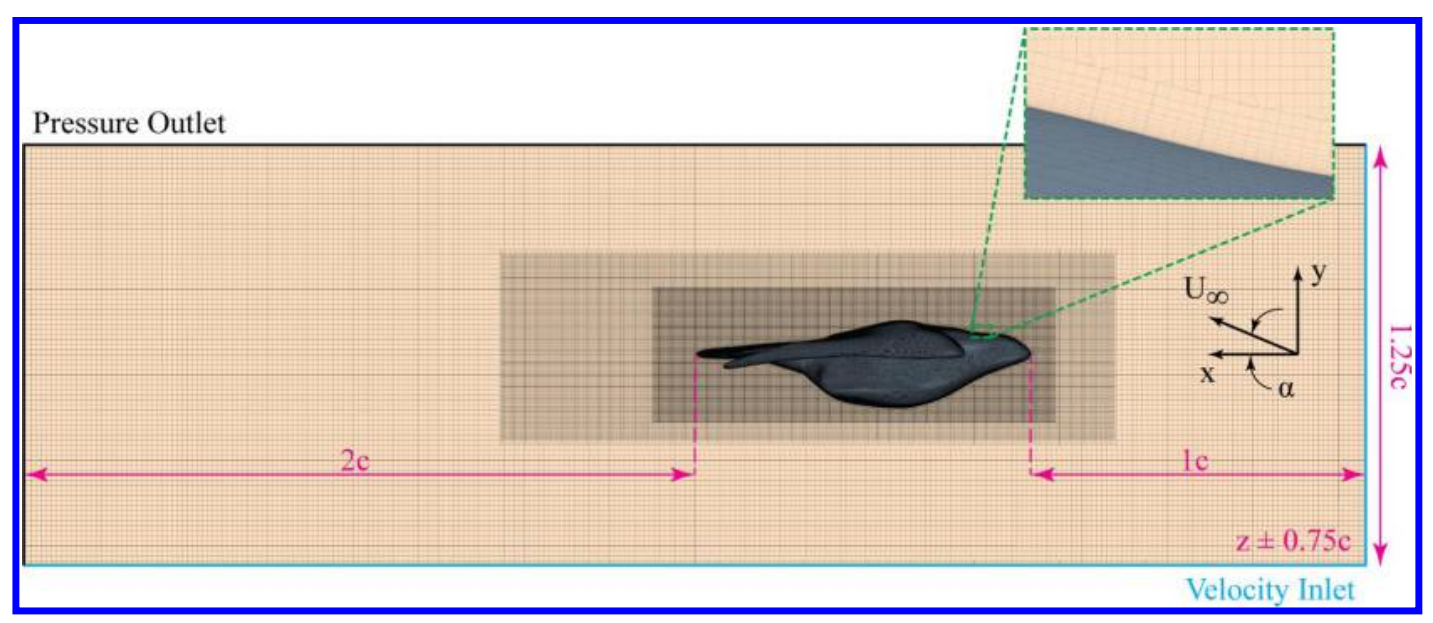

Fig. 3 CFD computational domain viewed in $x-y$ plane. Boundary conditions indicated as pressure outlet and velocity inlet. Walls of domain in $z$ plane not indicated but are both pressure outlets. Close-up view of near-wall mesh seen in green, dashed box.

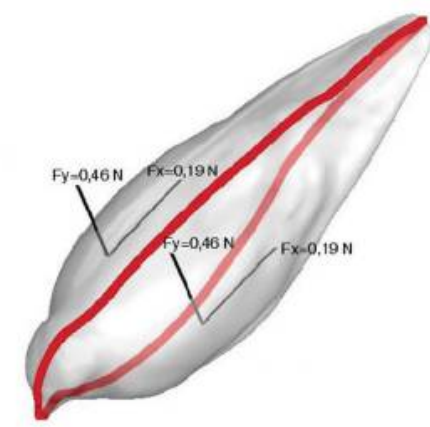

a) Teardrop shape

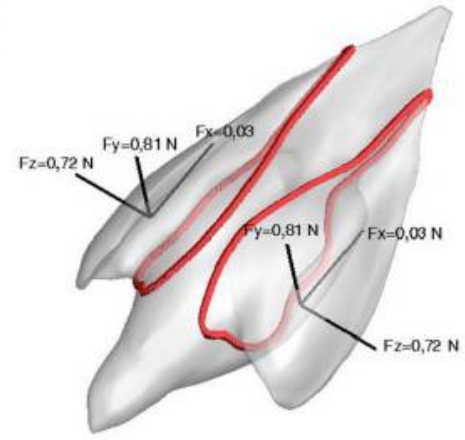

b) Cupped shape

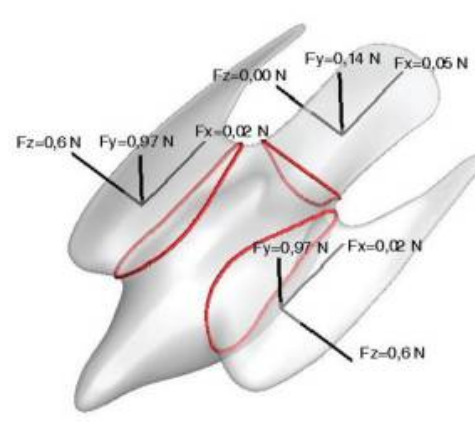

c) M shape

Fig. 4 Sectional aerodynamic forces over the falcon, the drag lift, and the side force represented as $F_{x}, F_{y}$, and $F_{z}$ respectively. All forces shown were taken at $\alpha=5$ deg.

middle of the cross section of the tunnel and mounted to a bespoke force balance via a sting. The force balance consists of three HBM PW15AH load cells, which have an accuracy of $0.02 \%$. The forces from the load cells can then be resolved such that the lift and drag can be obtained. More details on the experimental setup and the calibration of the system can be found in Ref. [5]. To compare with well established data, the forces had to be expressed in nondimensional forms, $C_{L}$ and $C_{D}$, by dividing by the corresponding sectional planform area $S$, the freestream dynamic pressure $q_{\infty}$

$$
C_{(x, y, z)}=\frac{F_{(x, y, z)}}{q_{\infty} S}
$$

Considering the forces acting over the horizontal and normal planes, the lift coefficient $C_{L}$ and drag coefficient $C_{D}$, respectively, can be expressed as functions of the axial and normal force coefficients $\left(C_{x}\right.$ and $\left.C_{y}\right)$ and the angle of incidence $\alpha$ :

$$
\begin{gathered}
C_{L}=C_{y} \cos \alpha-C_{x} \sin \alpha \\
C_{D}=C_{y} \sin \alpha+C_{x} \cos \alpha
\end{gathered}
$$

Further insight into the flow topology was obtained with the use of surface oil-flow visualization techniques. The oil-flow visualizations were carried out in a closed-loop low-speed wind tunnel at City, University of London. The mixture for the surface oilflow visualizations consisted of Day-Glo powder, mineral spirits (naphtha), and oleic acid. This was then applied to the model, using a paint brush, immediately before the wind tunnel was turned on. When the wind tunnel was turned on, the oil was evaporated by the airflow over the model, leaving the pigment behind on the surface of the model. The pigment that was left behind showed a "streaky" pattern, which shows the near-wall streamlines caused by the shear stress between the surface and the near-wall flow. Images were subsequently taken under ultraviolet lights in order to improve the contrast of the streaks.

\section{Results and Discussion}

\section{A. Static Aerodynamic Loads}

The falcon generates most of the lift while in the $M$ shape, which is the configuration adopted toward the end of the stoop, as shown schematically in Fig. 1. This would help it to start pulling out by pitching up immediately before or after striking the prey. From the field experiment by Ponitz et al. [5], while flying in the teardrop shape and cupped shape, during the dive, the falcon is normally flying at an angle of incidence of $\alpha<5 \mathrm{deg}$, and therefore generates very little lift. Figure $4 \mathrm{a}$ shows the sectional forces on the different configurations of the model produced by LES. From these, it can also be seen that the teardrop and cupped configurations generate considerably less left than the M-shape configuration. In these configurations, the requirement for lift is minimal. The bird only wants to achieve maximum speed while in the teardrop shape; in the cupped shape, it is either correcting its attitude to follow a moving prey, by changing the yaw or roll angle, or reducing its speed. This trend in lift force was also confirmed from the numerical simulation, which showed an excellent agreement with the lift measured experimentally on the M shape (see Fig. 5).

The agreement between the surface oil-flow visualization and the near-wall streamline pattern at $\alpha=5^{\circ}$ (seen in Fig. 6) confirms the reliability of the simulations. As reported previously in Ref. [7] and shown by the highly 3-D and inflectional surface shear-stress lines in Fig. $\underline{6}$, the flow over the falcon is dominated by vortical structures, 


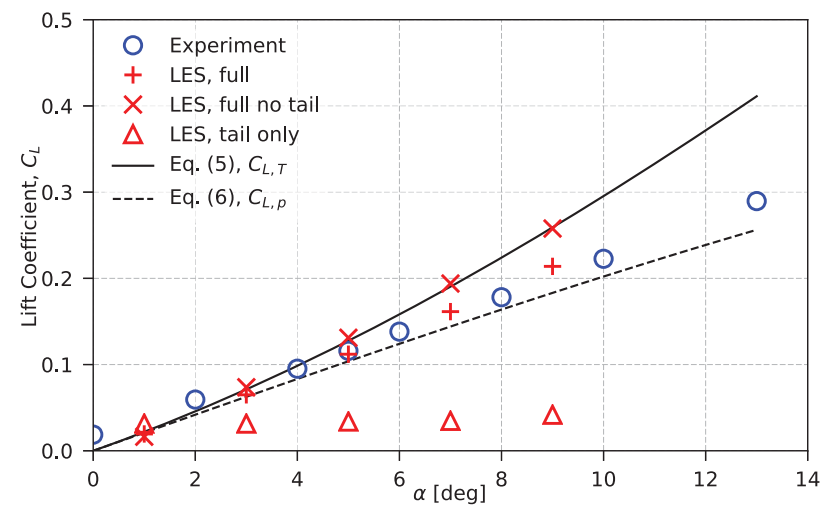

Fig. 5 Theoretical, experimental, and computational lift coefficient curves; confirmation that lift coefficient generated by wings only in $M$ shape agrees well with lift theory on delta wings.

even in the low-lift teardrop-shape and cupped-shape configurations. The linear characteristic of the total lift from the M shape in Fig. 5 encouraged further comparison with the well-established lift theory.

From the theory developed by Polhamus [14], in a subsonic flow, the total theoretical lift $C_{L_{T}}$ generated on a delta wing can be given as the sum of the potential flow lift $C_{L_{p}}$ and the contribution from the vortex flow $C_{L_{v}}$. Therefore,

$$
\begin{gathered}
C_{L_{T}}=C_{L_{p}}+C_{L_{v}} \\
C_{L_{p}}=K_{p} \sin \alpha \cos ^{2} \alpha \\
C_{L_{v}}=K_{v} \sin ^{2} \alpha \cos \alpha
\end{gathered}
$$

where $K_{p}$ and $K_{v}$ are the potential flow and vortex flow coefficients given as a function of the wing aspect ratio. For the comparison, these values were taken as $K_{p}=1.2$ and $K_{v}=\pi$, where the aspect ratio of an equivalent delta wing was taken as $A R=1$.

Since the flow over the bird was dominated by large vortices similar to those on delta wings, Polhamus's [14] theory for unsteady lift generation was preferred as opposed to the steady thin airfoil theory. In Fig. 5 (at first glance), at low incidence, the lift coefficient $C_{L_{T}}$ both measured and computed on the full model showed a closer agreement with the potential flow theory $C_{L_{P}}$. Further increase in $\alpha$ showed a deviation, but it was still not close enough to the theory for vortex lift. After subtracting the lift generated by the tail from the overall lift and following the appropriate nondimensionalization, the agreement with the vortex-lift theory in Fig. $\underline{5}$ is significantly improved.

Despite the formation of the strong vortices on the tail, the lift coefficient does not match the theory of vortex lift in Fig. 5; therefore, the tail does not act as a delta wing, which is an observation supported by previous investigations [15-17]. In fact, the tail generates lift even at a low angle of incidence at $\alpha \approx 0 \mathrm{deg}$. This ensures that it can be used for control during any flight conditions. From Fig. 6, especially on the $\mathrm{M}$ shape, it is important to point out that there is an upstream conditioning of the flow in the mid-dorsal region. This promotes a reattachment to ensure that the flow does not separate while progressing toward the tail, and hence high effectiveness of the tail is maintained and major deployment is not necessary. This has been reported in detail by Gowree et al. [7].

\section{B. Static Longitudinal Stability}

For comparison, the center of pressure (c.p.) was first built from the LES taking only the forces acting on one wing and compared to that built from the entire wing/body/tail combination (Figs. $7 \mathrm{a}$ and $8 \mathrm{~b}$ ). This allows conclusions to be drawn about the importance of the body/tail on the stability. The positively cambered wings will generate a negative "nosedown" pitching moment by their nature. The same is true for the body and tail of the bird because they are also liftproducing surfaces, which is contrary to tailplanes in most aircraft. The pitching moment about the aerodynamic center was found by tracking the movement of the c.p. at various incidences and taking the moment about various points until a location $x_{\mathrm{ac}}$ was found where the pitching moment stayed fairly constant. For the pitching moment around the center of gravity (c.g.), it follows that

$$
C_{m, \mathrm{c} . \mathrm{g}}=C_{m_{0}, \mathrm{ac}}+C_{y} \cdot\left(\frac{x_{\mathrm{c} . \mathrm{g}}-x_{\mathrm{ac}}}{c_{w}}\right)
$$

where $C_{m_{0} \text {,ac }}$ is the zero-lift pitching moment of the body about its aerodynamic center, and $C_{y}$ is the normal force coefficient of the body.

The criteria for static longitudinal stability, according to classical stability theory, are that there exists an appropriate angle of attack where the aircraft is in pitching moment equilibrium (trimmed flight), and that the pitching moment of an aircraft after departure from that equilibrium is always a restoring one. That is, the moment must be negative (pitchdown) following an upward disturbance and positive (pitchup) following a downward one. This can be quantified by inspecting the slope of the pitching moment curve at a trimmed condition (i.e., $C_{m, \text { c.g }}=0$ ). The condition is that the slope

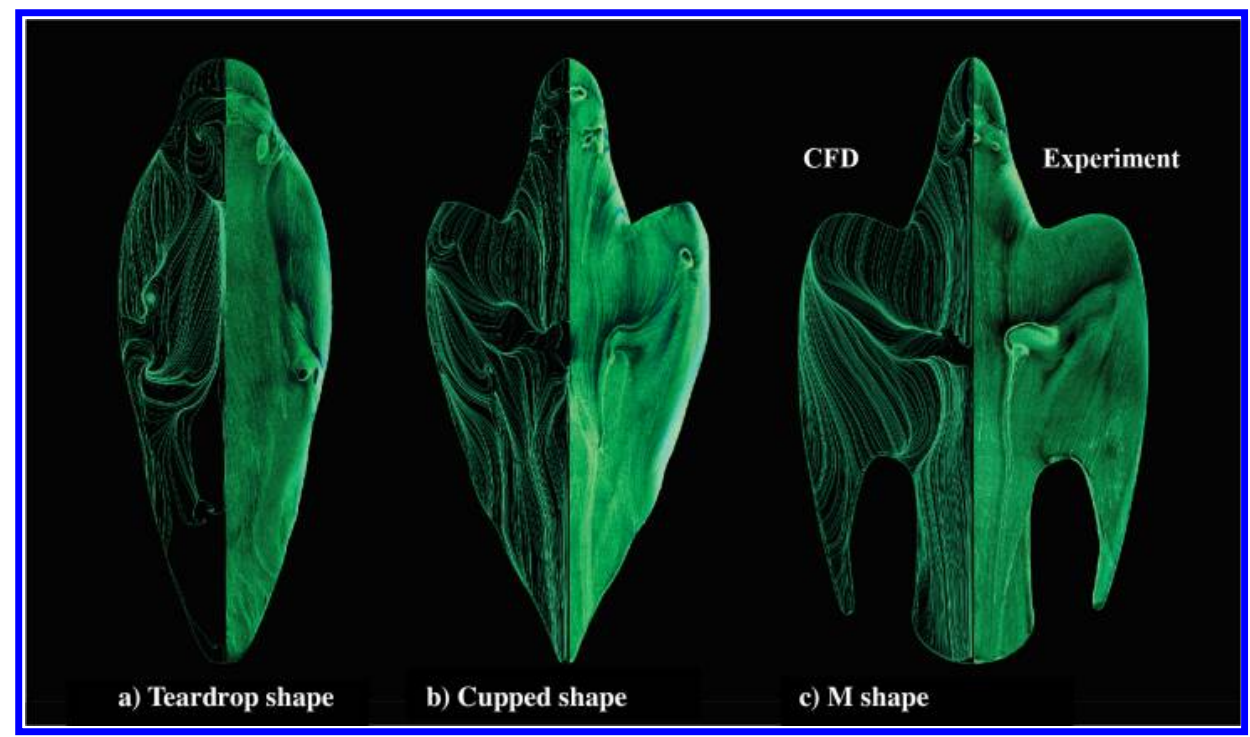

Fig. 6 Surface streamline patterns from oil-flow visualization experiments (right half) and skin-friction streak lines from LES (left half). All images taken at $\alpha=5$ deg. 


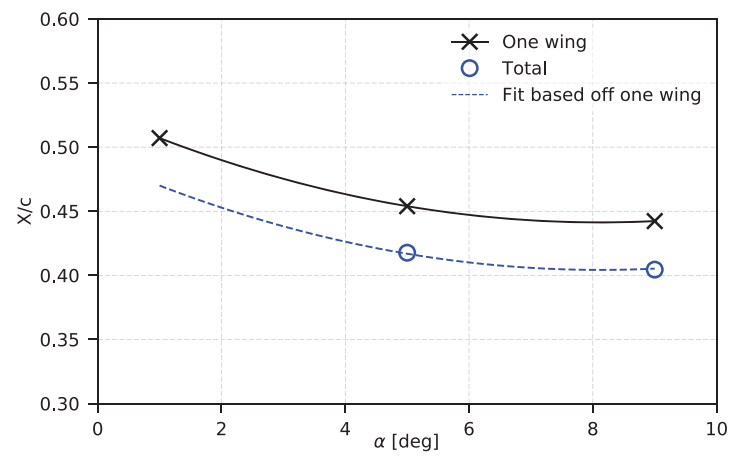

a) The c.p. location along the bird's longitudinal axis

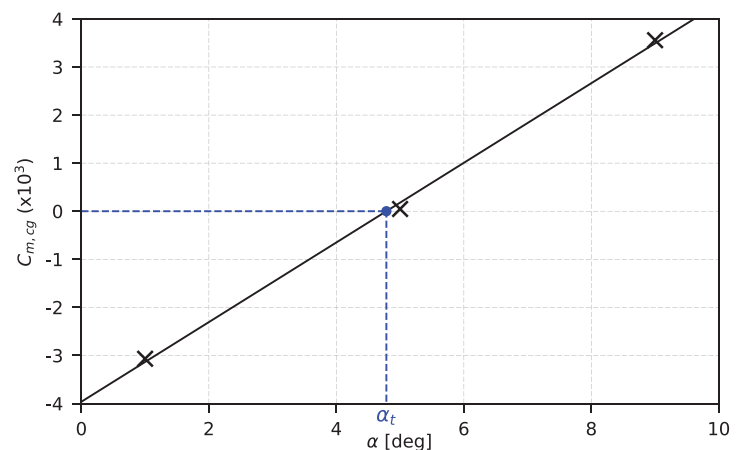

b) Pitching moment coefficient about the c.g. for the wing/body/tail combination, where $\alpha_{t}$ indicates the trim angle

Fig. 7 Center-of-pressure location and resulting pitching moment analysis.

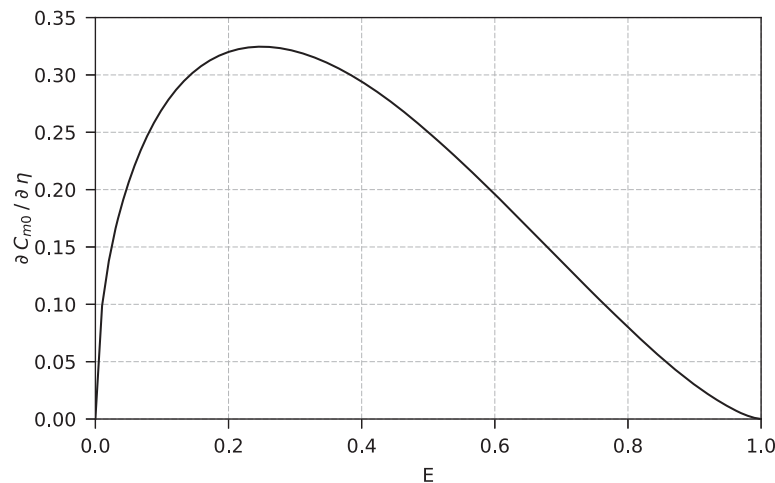

a)

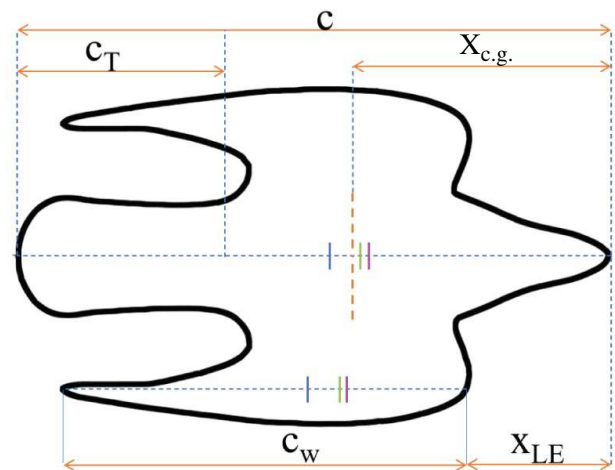

b)

Fig. 8 Representations of a) change in pitching moment coefficient $C_{M 0}$ per angle of flap deflection $\eta$ as given by Glauert [18]; and b) outline of model showing lengths and c.p. locations, at $\alpha=1,5$ and $9 \mathrm{deg}$ respectively from left to right.

be negative. That is, it must be at an incidence of $\alpha=\alpha_{t}, C_{m, \mathrm{c.g}}=0$, and

$$
\frac{d C_{m, \mathrm{c.g}}}{d \alpha}<0
$$

Analyzing the forces and moments from the LES results can give an insight into the longitudinal stability of the falcon in such a maneuver. The center of pressure for one wing shows, as expected, a steady movement toward the leading edge with the increase in incidence. Similarly, this was seen in the entire bird's center of pressure. However, the point at $1 \mathrm{deg}$ was shown to be an outlying point; subsequently, using the fit based on the wing c.p. movement, the point at $1 \mathrm{deg}$ was estimated as shown in Fig. $7 \mathrm{a}$.

Using Eq. (7), the pitching moment about the c.g. of the model was plotted (Fig. $\overline{7 b}$ ) and shown to have a marginal positive (unstable) slope. The trim point was found at $\alpha_{t} \approx 5 \mathrm{deg}$. This is close to the angle of attack observed in peregrine falcon stooping flight at the start of the pullout [5]. This is the angle of attack at which, at the tail setting of the model, the bird would aim to fly in order to maintain balanced equilibrium of moments about the c.g. The trim point is usually adjusted by tail morphing, and this reflects that of the current (nominal) tail position.

The limited use of the tail for such adjustments at high speed could also be attributed to its high effectiveness such that small deflections can produce large pitching moments without the need to fully deploy; this is due to the ratio of the tail-to-body length, acting as a trailingedge flap. For a plain-flap airfoil, the change in pitching moment coefficient $C_{M 0}$ per angle of flap deflection $\eta$ is illustrated in Fig. 8a and was given by Glauert [18] as

$$
\frac{\partial C_{m 0}}{\partial \eta}=\sqrt{E(1-E)^{3}}
$$

where $E$ is the flap-to-chord ratio for an airfoil. Considering the tail as a trailing-edge flap, the tail length of the falcon model $C_{T}$ is approximately just over one-third of its body length $C$ (see Fig. 8b, as per the sectional forces taken in Fig. 4c). This would correspond to a value of $E=0.35$ in Fig. 8a, where it is most effective (near the maximum at the curve). This natural sizing of optimized trailing-edge morphing surfaces was also found in aquatic life in hydrodynamic analysis of a rainbow trout (Oncorhynchus mykiss) $[19,20]$. It was found by Song et al. [21] that barn owls use their tails as "trailing-edge" control surfaces by applying downward tail deflection for lift production to offset the loss of continuous spanwise lift over the body, and therefore reduce induced drag. The tail-length-to-body-length ratio of barn owls is $\approx 0.38$ for adults [22], which is slightly larger than that of the peregrines but still within the highly effective area. Further work focused on the role and effectiveness of birds' tails in fast flight is left as future work.

It is apparent that, in a pullout maneuver, the falcon will fly in a marginal longitudinal static instability. This could be to exploit the benefits in maneuverability in addition to eliminating trim drag, which is the portion of drag associated with lift produced to counter tail balancing in stable aircraft.

\section{Loads During Pullout}

The pullout for an ideal falcon was defined by Tucker [3] as the phase in which it follows a circular arc until the glide path is horizontal. In reality, this path is a series of arcs decreasing in radius as the bird nears horizontal. By taking a centrifugal force balance about the center of the instantaneous circular pullout arc, it can be shown that in order to sustain the maneuver, the lift is equal to the sum of the centrifugal force and the component of weight radial to the pullout (i.e., normal to the flight direction).

Using the instantaneous pitching angle $\theta$ and velocity $U$ from the recordings [5] for the weight of the bird in question, the load factor $n$ can be obtained via Eq. (10), and subsequently the aerodynamic loads: 


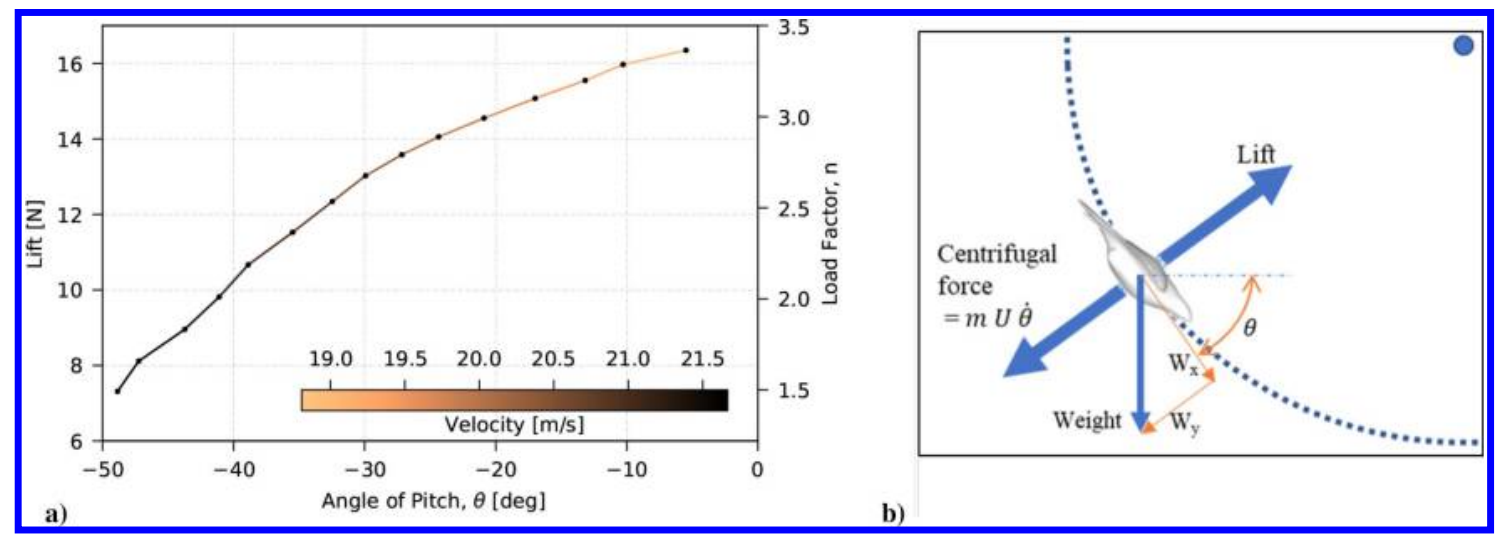

Fig. 9 Representations of a) lift and load factor throughout a pullout maneuver, and b) centrifugal force balance during a pullout. These were obtained by using Eq. (10) and considering values reported from field experiment by Ponitz et al. [ㅁ].

$$
n=\frac{U \dot{\theta}}{g}+\cos (\theta)
$$

In Fig. 9a, a gradual increase in lift is observed throughout the maneuver as the bird tightens its pullout radius and approaches level flight. However, the velocity can be seen to undergo a marginal decrease to prevent the bird from stalling or allowing it to go around for another strike in case it misses the prey. In this case, a maximum load factor of $3.2 \mathrm{~g}$ was estimated despite the field experiment being conducted on a trained falcon in a controlled environment, where the maximum speed attained was limited by the altitude of the dam in Ref. [5].

The velocity attained during the field experiment in Ref. [5] was significantly lower than that normally observed on wild falcons in stoop, which seems to be the highest during courtship stoop demonstrations. $\#$ Peregrine falcons can reach velocities of $80 \mathrm{~ms}^{-1}$; by setting this as the initial velocity for the pullout (i.e., the velocity at equilibrium) and using the same methods as before, the bird will produce up to a maximum of $47 \mathrm{~N}$ of lift at the tightest part of the pullout.

\section{Wing Morphing During Pullout}

In the previous section, the lift generated by the bird during the pullout was calculated based on the glide path and speed of the bird during the field experiment. After nondimensionalization, from Fig. 10a, the maximum $C_{L}$ reached in the $\mathrm{M}$ shape was twice as high as that measured during the wind-tunnel test or computed from the LESs, even at high incidence. To attain such high $C_{L}$ while maintaining the $\mathrm{M}$ shape, the bird had to be flying at very high incidence, which would increase the form drag significantly; but, Fig. 9 confirms that the reduction in speed in this phase was marginal. To attain such a high lift coefficient without significant increase in drag, the bird was seen to modify its wing morphology during the pullout, following the trends represented by the sequence of silhouettes in
Fig. 10a, which was confirmed from the live recordings during the field experiments. Here, the main morphological transformation occurs through the sweep angle change of the primaries, which would result in an increase in span and lifting surface. While reducing the sweep angle of $\Lambda_{\max }=90 \mathrm{deg}$ to $\Lambda_{\min }=40 \mathrm{deg}$ (morphing from M-shape to Open-M) toward the end of pullout, the aspect ratio $A R$ for the model is increased from approximately 0.95 to 4.9 , respectively. Earlier, it was demonstrated that the lift generation by the wings was similar to that over delta wings. Extension of the delta wing theory here for a range of aspect ratios (Fig. 10b) suggests that in order to achieve maximum required $C_{L}$ toward the end of pullout while operating at moderate to low angles of incidence, an aspect ratio tending toward five would at least be required. Further work is suggested to verify this via LES and wind-tunnel tests on the model at larger-aspect-ratio configurations. From Fig. 10b, it can be seen that as the aspect ratio approaches $A R=4$, the curves seem to converge. Hence, it can be concluded that when $A R>4$, the $C_{L}$ curves will collapse onto one another.

Although the increase in lift is achieved through in-plane symmetrical morphing (change in sweep) of primary feathers moving from the $\mathrm{M}$ shape toward fully deployed primaries, both in-plane and outof-plane asymmetrical morphing provides yaw control coupled with roll through a bank turn in the absence of a vertical tailplane, unlike in most aircraft. This asymmetrical morphing is also observed during other phases of the stoop when the bird is readjusting its trajectory. Pure yaw control in the cupped shape can be achieved due to the substantial amount of side force generated on the wings, as shown in Fig. 4 (see also Ref. [8]), as well as by the strong vortices that are now aligned to the side of the bird. This side force does not degrade significantly while morphing into the $M$ shape, hence allowing the bird to engage easily into a yaw maneuver if it needs to turn around for another attempt.

Lacking vertical stabilizers, birds need to continuously augment their yaw stability by twisting their tails [23]; faster birds need larger tails for this purpose, as seen in swifts and hawks [10]. This element
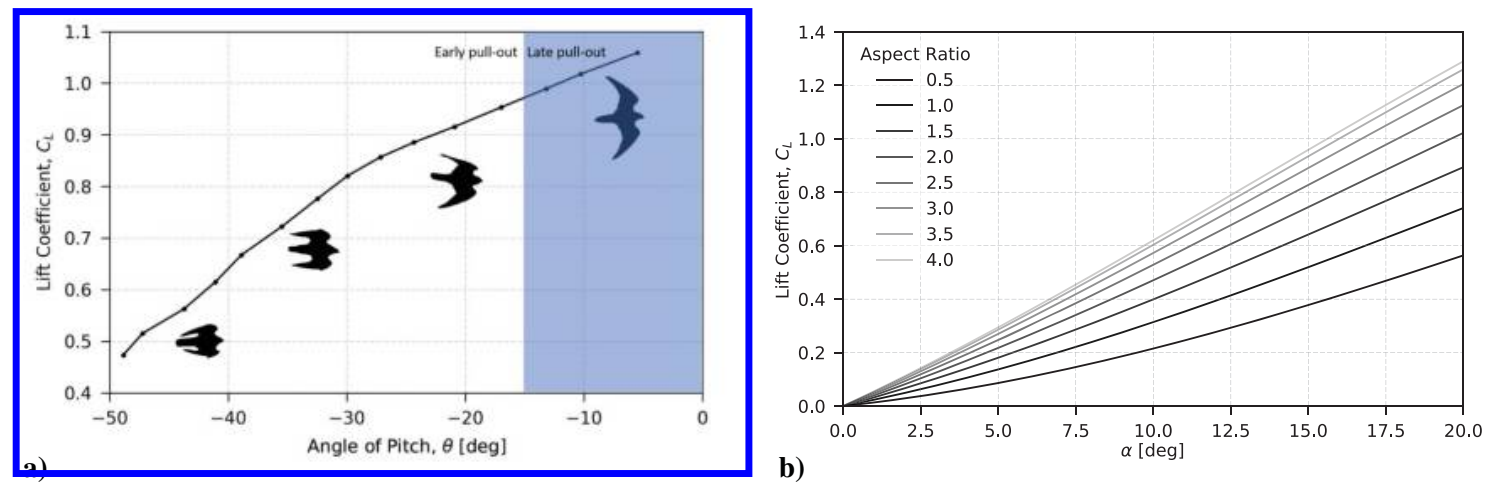

Fig. 10 Representations of a) lift coefficient in pullout illustrating different configurations, with $M$ shape in the early pullout to open $M$ at $\Lambda_{\text {min }}$ at the end of pullout; and b) effect of varying the aspect ratio $A R$ on the theoretical lift coefficient. 
of side force generated by the out-of-plane morphing can offer an element of yaw control in a crabbed turn in addition to possibly augmenting yaw stability via asymmetric morphing, reducing the load and the associated size constraint from the tail in a pullout or eliminating its need entirely. These vortices are the main contributors to the aerodynamic forces and play an important role in the maneuverability of the falcon, where the pitch, yaw, or roll could be achieved simply by morphing and projecting the wing over different planes.

\section{Conclusions}

The present work describes experimental measurements and highresolution CFD simulations of the flow and aerodynamic forces on a peregrine falcon during the sequence of geometrical changes when the bird pulls out of a high-speed stoop maneuver. From the LES data, the pressure field was obtained on the highly complex three-dimensional geometry of the bird and wings with strong curvature in all principle directions of the body. This allowed determination of the center of pressure and the moment coefficient $C_{m}$, which would otherwise be difficult to obtain from experiments for such a complex body. Validation of the LES was done using integral force-balance measurements, oil-flow visualization of the surface streamlines, and particle image velocimetry measurements in the wake of the models at the same boundary conditions as in the simulation (see the work of Gowree et al. [7]). The results are limited to the early pullout phase until the $\mathrm{M}$ shape because no exact geometrical data for the late stage of the open $\mathrm{M}$ are available. It is seen from the presented results that the principle features of the near-wall flow topology do not change when morphing from the cupped configuration $(A R=0.5)$ to the $\mathrm{M}$ shape $(A R=1)$, as inferred from Fig. $\underline{6}$. Therefore, the same vortical structures are expected to persist for increasing aspect ratios (open-M configuration): perhaps while different in strength. As the latter is essentially the morphing of the hand wings to smaller sweep angles, aerodynamic lifting theory is used to predict the further extension toward this late stage.

An assessment of the static aerodynamic loads generated by the bird in the $\mathrm{M}$ shape showed that the lift generated purely by the wing matches very well with Polhamus's theory [14], and this is a result of the formation of large vortices, similar to that on delta wings. The extension of the delta-wing theory allowed for further hypothesis on the flight mechanics of the bird toward the end of the stoop or pullout. The static longitudinal stability analysis confirmed that, in the Mshape configuration, the bird was flying unstably in pitch, based on the positive slope in the pitching moment coefficient. This allows the falcon to fly in a responsive and maneuverable fashion and in a trajectory with minimal loss in energy and forward velocity. Further analysis of the pullout maneuver showed that during the controlled field experiment of Ponitz et al. [5], the bird would be experiencing a load factor of approximately $3 . \overline{5} \mathrm{~g}$, but that can be tripled during stoops in wild conditions.

Analysis of the wing morphing showed that the high lift required toward the end of pullout was achieved by forward sweeping of the primary feathers while maintaining the angle of incidence as low as possible for lower drag. Asymmetrical and out-of-plane morphing of the primaries in the form of dihedral change will also provide yaw and roll control without much input from the tail. The furling of the tail in $M$ shape suggests that this control is handled via instantaneous morphing of the wings, i.e., changing the sweep/aspect ratio to alter the amount of lift and pitching moment to match the requirement, reducing or eliminating the need for large tail inputs and their associated increase in drag. The minimal requirement of tail inputs for longitudinal adjustments was also attributed to its high effectiveness; this is due to the natural optimization of the tail-to-body length, which is also observed in other avian and aquatic species.

Some quantitative studies have been provided herein on how a falcon can achieve its superior maneuverability through wing morphing from live-flight observations and measurements during field experiments, wind-tunnel testing, and numerical simulation. These findings can be extrapolated for the design of convertible bioinspired
UAVs and MAVs, or even more versatile and highly maneuverable fixed-wing aircraft.

Incorporating this in bioinspired UAV/MAVs could be realized with vortex-lift/morphing-wing aircraft controlled by a lightweight FCC, as well as a suite of sensors designed to detect departures from equilibrium and respond actively and instantaneously with simple wing morphing to maintain desired attitude in a pullout maneuver or any similar maneuver. To design this, more needs to be known about the effect of altering the sweep and aspect ratio on leadingedge vortex-lift production and the center-of-pressure location for small-scale high-speed morphing-wing aircraft and natural fliers at Reynolds numbers of $\approx 10^{5}$ to $10^{6}$. The benefits of adopting nature-inspired control alternatives are a possible reduction in the number of control surfaces, their weights, response times, and their associated increase in power consumption and overall drag.

\section{Acknowledgments}

The position of Christoph Brücker is cofunded by BAE Systems and the Royal Academy of Engineering (research chair no. RCSRF1617\4\11), and the position of Omar Selim is funded by the George Daniels Educational Trust: all of which are gratefully acknowledged. The authors express profound thanks to T. Colditz and M. Gelfert by whom the force measurements were carried out on the different falcon models in 2013 as part of their studies at Technische Universität Bergakademie Freiberg. In addition, we thank Mike Newsam from Stellar Advanced Concepts, Ltd. for his fruitful discussions on bioinspired wing-morphing concepts. The authors would also like to acknowledge the support provided by the U.K. National Wind Tunnel Facility to carry out the flow visualization experiments at City, University of London. O. Selim is credited with conceptualization, methodology, validation, formal analysis, investigation, data curation, writing-original draft, writing-review and editing, visualization, and project administration; E. R. Gowree is credited with conceptualization, validation, formal analysis, investigation, writing-original draft, and writing-review and editing; C. Lagemann is credited with methodology, software, validation, formal analysis, investigation, data curation, writingoriginal draft, writing-review and editing, and visualization; E. Talboys is credited with methodology, validation, investigation, data curation, writing-original draft, writing-review and editing, and visualization; C. Jagadeesh is credited with methodology, validation, investigation, writing-original draft, and writingreview and editing; and C. Brücker is credited with conceptualization, methodology, writing-original draft, writing-review and editing, supervision, and funding acquisition.

\section{References}

[1] Alerstam, T., "Radar Observations of the Stoop of the Peregrine Falcon Falco Peregrinus and the Goshawk Accipiter Gentilis," Ibis, Vol. 129, Jan. 1987, pp. 267-273. https://doi.org/10.1111/j.1474-919X.1987.tb03207.x

[2] Tucker, V. A., Cade, T. J., and Tucker, A. E., "Diving Speeds and Angles of a Gyrfalcon (Falco rusticolus)," Journal of Experimental Biology, Vol. 201, No. 13, 1998, pp. 2061-2070. https://doi.org/10.1242/jeb.201.13.2061

[3] Tucker, V. A., "Gliding Flight: Speed and Acceleration of Ideal Falcons During Diving and Pull Out," Journal of Experimental Biology, Vol. 201, No. 3, 1998, pp. 403-414

https://doi.org/10.1242/jeb.201.3.403

[4] Schmitz, A., Ondreka, N., Poleschinski, J., Fischer, D., Schmitz, H., Klein, A., Bleckmann, H., and Bruecker, C., "The Peregrine Falcon's Rapid Dive: On the Adaptedness of the Arm Skeleton and Shoulder Girdle," Journal of Comparative Physiology A, Vol. 204, No. 8, 2018, pp. 747-759.

https://doi.org/10.1007/s00359-018-1276-y

[5] Ponitz, B., Schmitz, A., Fischer, D., Bleckmann, H., Brücker, C., and Aegerter, C. M., "Diving-Flight Aerodynamics of a Peregrine Falcon (Falco peregrinus)," PLOS ONE, Vol. 9, No. 2, 2014, Paper e86506. https://doi.org/10.1371/journal.pone.0086506

[6] Durston, N. E., Wan, X., Liu, J. G., and Windsor, S. P., "Avian Surface Reconstruction in Free Flight with Application to Flight Stability Analysis of a Barn Owl and Peregrine Falcon," Journal of Experimental 
Biology, Vol. 222, No. 9, 2019, Paper jeb185488. https://doi.org/10.1242/jeb.185488

[7] Gowree, E. R., Jagadeesh, C., Talboys, E., Lagemann, C., and Brücker, C., "Vortices Enable the Complex Aerobatics of Peregrine Falcons," Communications Biology, Nature, Vol. 1, No. 1, 2018, pp. 1-7.

[8] Ponitz, B., Triep, M., and Brücker, C., "Aerodynamics of the Cupped Wings During Peregrine Falcon's Diving Flight," Open Journal of Fluid Dynamics, Vol. 4, No. 4, 2014, pp. 363-372. https://doi.org/10.4236/ojfd.2014.44027

[9] Tucker, V. A., and Parrott, G. C., "Aerodynamics of Gliding Flight in a Falcon and Other Birds," Journal of Experimental Biology, Vol. 52, No. 2, 1970, pp. 345-367. https://doi.org/10.1242/jeb.52.2.345

[10] Brown, R., "The Flight of Birds," Biological Reviews, Vol. 38, No. 4, 1963, pp. $460-489$. https://doi.org/10.1111/j.1469-185X.1963.tb00790.x

[11] Tucker, V. A., "Pitching Equilibrium, Wing Span and Tail Span in a Gliding Harris' Hawk, Parabuteo Unicinctus," Journal of Experimental Biology, Vol. 165, No. 1, 1992, pp. 21-41. https://doi.org/10.1242/jeb.165.1.21

[12] Meyers, J., Geurts, B., and Sagaut, P., Quality and Reliability of LargeEddy Simulations, Vol. 12, Springer Science and Business Media, New York, 2008, pp. 181-184.

[13] Lagemann, C., Gowree, E., Jagadeesh, C., Talboys, E., and Brücker, C., "Experimental and Numerical Analysis of the Aerodynamics of Peregrine Falcons During Stoop Flight," Deutsche Gesellschaft für Luftund Raumfahrt - Lilienthal-Oberth e.V., 2018. https://doi.org/10.25967/480311

[14] Polhamus, E. C., "Predictions of Vortex-Lift Characteristics by LeadingEdge Suction Analogy," Journal of Aircraft, Vol. 8, No. 4, 1971, pp. 193-199. https://doi.org/10.2514/3.44254

[15] Maybury, W. J., Rayner, J. M. V., and Couldrick, L. B., "Lift Generation by the Avian Tail," Proceedings of the Royal Society of London, Series
B: Biological Sciences, Vol. 268, No. 1475, 2001, pp. 1443-1448. https://doi.org/10.1098/rspb.2001.1666

[16] Evans, M. R., Rosén, M., Park, K. J., and Hedenström, A., "How Do Birds' Tails Work? Delta-Wing Theory Fails to Predict Tail Shape During Flight," Proceedings of the Royal Society of London, Series B: Biological Sciences, Vol. 269, No. 1495, 2002, pp. $1053-$ 1057.

[17] Evans, M. R., "Birds' Tails do Act Like Delta Wings but Delta-Wing Theory Does Not Always Predict the Forces they Generate," Proceedings of the Royal Society of London, Series B: Biological Sciences, Vol. 270, No. 1522, 2003, pp. 1379-1385.

[18] Glauert, H., "Theoretical Relationships for an Aerofoil with Hinged Flap," British Aeronautical Research Committee R\&M 1095, 1927.

[19] Przybilla, A., Kunze, S., Rudert, A., Bleckmann, H., and Brücker, C., "Entraining in Trout: A Behavioural and Hydrodynamic Analysis," Journal of Experimental Biology, Vol. 213, No. 17, 2010, pp. 29762986. https://doi.org/10.1242/jeb.041632

[20] Brücker, C., "Dynamic Interaction in Bluff Body Wakes," Professional Dissertation, RWTH Aachen, Shaker Verlag, 2006.

[21] Song, J., Cheney, J. A., Usherwood, J. R., and Bomphrey, R. J., "Virtual Manipulation of Bird Tail Postures Demonstrates Drag Minimisation when Gliding," BioRxiv, 2020. https://doi.org/10.1101/2020.06.09.141994

[22] Marti, C. D., "Sex and Age Dimorphism in the Barn Owl and a Test of Mate Choice," Auk, Vol. 107, No. 2, 1990, pp. 246-254. https://doi.org/10.2307/4087606

[23] Thomas, A. L., and Taylor, G. K., "Animal Flight Dynamics I. Stability in Gliding Flight," Journal of Theoretical Biology, Vol. 212, No. 3, 2001, pp. 399-424.

https://doi.org/10.1006/jtbi.2001.2387
H. Dong Associate Editor 